\title{
What colour is penguin guano?
}

\author{
W. G. Rees ${ }^{1}$, J. A. Brown ${ }^{1,2}$, P. T. Fretwell ${ }^{2}$, P. N. Trathan ${ }^{2}$
}

1: Scott Polar Research Institute, University of Cambridge, Lensfield Road, Cambridge CB2 1ER, UK.

2: British Antarctic Survey, Madingley Road, Cambridge CB3 0ET, UK.

\begin{abstract}
The identification and quantification of Antarctic Pygoscelis penguin colonies depends increasingly on recognition of the characteristic optical properties of guano deposits, but almost all knowledge of these properties until now has been compromised by resolution and atmospheric propagation effects. Here, we present hyperspectral reflectance data in the range 350-2500 nm, collected in situ from fresh guano deposits in Pygoscelis penguin colonies on Signy Island, South Orkney Islands. The period of data collection included the transition from predominantly white guano to the pink coloration characteristic of a krill-rich diet. The main identifiable features in the spectra are a broad absorption feature centred around $550 \mathrm{~nm}$, responsible for the pink coloration and identified with the pigment astaxanthin, as well as several water absorption features. Variations in these features are responsible for differentiation between spectra. From these results we propose two spectral indices, suitable for use with satellite data, one of which responds to the presence of astaxanthin in the guano and the other to water. Our results do not allow us to differentiate between penguin species from their guano, but do suggest that the breeding phenology of Pygoscelis penguins could be determined from a time-series of multispectral imagery.
\end{abstract}

\section{Introduction}

ecosystem.

Given their role in ecosystem monitoring and It is important to be able to monitor penguins in the management, accurate, unbiased and comprehensive Antarctic, both to enhance our understanding of assessments of the populations of these three penguin Southern Ocean ecology (Trathan et al. 2015) and species are of key management and conservation also more generally as indicators of environmental importance. The best opportunity to monitor penguin variability and change (Boersma 2008, Forcada \& numbers is when they come ashore to breed. GroundTrathan 2009, Weimerskirch et al. 2003). Three of the based monitoring programmes with standard six penguin species that breed in the Antarctic are methodologies and protocols have been developed Pygoscelis penguins (Croxall \& Kirkwood 1979): (CCAMLR 1992, Croxall \& Kirkwood 1979, Agnew Adélie (Pygoscelis adeliae), gentoo (Pygoscelis 1997), but these approaches are logistically papua) and chinstrap penguins (Pygoscelis expensive, of limited extent, and geographically antarctica). These species are numerous, and are key biased (LaRue et al., 2014). For example, regular consumers within Southern Ocean foodwebs, in monitoring occurs on the west Antarctic Peninsula particular of Antarctic krill (Euphausia superba), one where more research is focussed, compared with of the main prey species also utilized by a broad guild along the east Antarctic coast (Southwell et al. 2015), of fish, squid, seabirds and marine mammals. Because with an estimated 10-15\% of all Adélie colonies of their sensitivity to variation in krill stocks, these monitored at least occasionally (LaRue et al. 2014). It penguin species are all considered to be valuable is highly unlikely that all, or even the majority, of indicators of environmental variability and change colonies will ever be visited regularly for direct and thus of ecosystem status (Ainley 2002). Indeed, monitoring through ground counts. These groundthe Commission for the Conservation of Antarctic based methods also have the potential to disturb Marine Living Resources (CCAMLR), which colonies because of observer activities and manages fisheries in the Southern Ocean, including procedures. Aerial photography can extend the the fishery for Antarctic krill, has developed an coverage of ground-based programmes (Trathan ecosystem monitoring programme (Agnew 1997) that 2004), but it remains logistically difficult. Remote uses the three Pygoscelis penguin species as sensing methods are thus attractive, especially those indicators of the status of the Antarctic marine based on the analysis of satellite imagery as they offer 
extensive coverage and minimal disturbance.

guano, which appears to be sufficiently distinctive

Recently a number of remote sensing studies that it can be recognised even when it is mixed with have estimated penguin numbers using satellite the spectra of other materials. Until recently the imagery collected over regional or continental scales spectral range of these very high resolution (VHR) (Fretwell et al. 2015, Fretwell \& Trathan 2009, Lynch sensors has been limited to the visible and near infra\& LaRue 2014, Schwaller et al. 2013). Although red wavelengths. The signature of guano at these these surveys have increased our knowledge about the wavelengths is not spectrally unique and so it is populations of penguins in Antarctica a number of difficult to identify colonies automatically, without challenges associated with these methods still remain. substantial manual intervention, though VNIR These include a better understanding the variability of imagery can be used to determine colony size where adult numbers on the ground at the time of image the location is already known. In particular, it appears acquisition and the relationship between the area of that it may be unique in the short wave infra-red guano staining and of penguins in the image and the (Fretwell et al. 2015).

total breeding population (Fretwell et al. 2015, Lynch The spectral distinctiveness of guano-covered et al. 2012, Lynch \& LaRue 2014, Southwell et al. areas is most apparent when the diet is rich in krill 2015). The use of satellite imagery for finding, and the guano is visually a reddish-brown colour, and counting and monitoring penguin populations has till this has been successfully exploited using pixel-based now mostly used medium resolution (30 metres) and super-pixel classification algorithms to Landsat imagery (Schwaller et al. 2013, Schwaller et differentiate guano (LaRue et al. 2014, Lynch \& al. 1989, Schwaller et al. 1984). However, the limited LaRue 2014, Waluda et al. 2014, Witharana \& Lynch spatial resolution of the Landsat data has inherent 2016). The transition from white to pink guano is problems differentiating between seabird groups and strongly related to the phase of the breeding cycle identifying sub-colonies and smaller breeding areas (Trathan, P.N., pers. comm.) so the ability to track this (Fretwell et al. 2015, Schwaller et al. 2013). As a transition reliably would have valuable use. Another consequence, more recent studies have primarily potential source of error when using remote sensing is focussed upon higher resolution sensors. Several the changing guano colour throughout the different studies have now utilized recently available very high stages of the breeding season, particularly with regard resolution satellite data to estimate Pygoscelis to the start of the season where the guano has yet to penguin numbers (LaRue et al. 2014, Lynch et al. 'pink up' so may not be distinct from the background 2012, Lynch \& LaRue 2014, Naveen et al. 2012, (such as snow) in satellite images and at the end of Waluda et al. 2014). Despite recent improvements in the season after crèching where denudation of guano the available spatial resolution, even the very highest makes colony boundaries indistinct in imagery resolution satellite imaging systems - which are now (Lynch et al. 2012). Conversely, as different species approaching or have exceeded resolutions of 1 metre have varying breeding phenologies, the application of - cannot reliably resolve individual Pygoscelis carefully timed satellite acquisition may be utilized to penguins. Even at this very high but still identify different species (Waluda et al. 2014). Since comparatively limited spatial resolution the there is some reason to believe that penguins pack at geographical coverage available from a single fairly constant species-specific areal density in satellite view is rather small - typically of the order of breeding colonies (Woehler \& Riddle 1998) and their $10 \mathrm{~km}$ - which is unfit for wide-scale studies. area can be used as an analogue of nesting population Therefore, a combination of low-resolution imagery (Schwaller et al. 1989, Schwaller et al. 2013), covering a broad area, coupled with high-resolution knowing the area that is in active use in a breeding imagery for specific colonies, appears to be a sensible colony implies some knowledge of the number of approach for the future. Consequently, this implies birds (although this will almost certainly also depend that the 'mixed pixel problem', in which the on species and on the type of terrain). Differences in individual pixels of the image contain mixtures of coloration at different times of year are a potential target materials including penguins themselves but source of error in current methods, though also have also various kinds of background materials, will potential for separating between species.

continue to need to be considered.

If the data defining representative spectra for

Methods of identifying penguin colonies in guano-covered terrain are derived from satellite satellite imagery have generally relied on identifying images (as is almost entirely true to date), they have and mapping areas of guano-covered ground (Waluda limited spectral resolution and they also represent et al. 2014, LaRue et al. 2014). These methods mixtures of guano and other materials, even at the therefore identify the guano stained areas based upon highest spatial resolutions so far attained. the spectral signature (i.e. the variation of reflectance Furthermore, they are difficult to correct for variable with wavelength across the visible and reflective atmospheric propagation effects by which infrared regions of the electromagnetic spectrum) of atmospheric gases and aerosols modify the amount of 
radiation detected at the top of the atmosphere the South Orkney Islands (fig. 1), during the period relative to what was reflected from the surface into November 2014-January 2015. Signy is a small (ca $7 \times$ the bottom of the atmosphere. All three factors mean $5 \mathrm{~km}$ ), largely ice-covered island on which all three they are difficult to generalise from one observing species of Pygoscelis penguins breed. Two of the system to another. Waluda et al. (2014) used four authors (WGR, JAB) were based at the British band QuickBird2 imagery to automatically classify Antarctic Survey's Signy Research Station (60.709 S, different species of penguins on Signy Island, South 45.595 W) during this period. All the measurements Orkney Islands. The study used remote sensing and described here were taken of samples of penguin GPS data without reference ground spectra and guano, or of penguin vomit, taken as being indicated that a better knowledge of phenological representative of the food being consumed within the changes in guano coloration and related breeding colony. We collected data from various sites around phenology would lead to more robust results. With the island, but mainly from the extensive colonies of the recent and planned launch of a number of Adélie and chinstrap penguins located on the Gourlay satellites that improve the spatial resolution, spectral Peninsula $(60.729 \mathrm{~S}, 45.586 \mathrm{~W})$. The period of data resolution and availability of satellite data collection included most of the relevant breeding (WorldView3, Sentinel 2+2a, PRISMA, ENmap and events for these two species, although the transition to HyspIR), potential advances in image analysis predominantly pink guano was complete only for techniques become possible.

Adélies (fig. 2). Samples relating to gentoo penguins

The aim of the work presented here is thus to were mostly collected near North Point $(60.672 \mathrm{~S}$, identify pure guano spectra, unmixed with other $45.626 \mathrm{~W})$ and Cemetery Bay $(60.706 \mathrm{~S}, 45.603 \mathrm{~W})$. background materials, uncontaminated by Geographical coordinates are given in the WGS 84 atmospheric propagation effects, and at sufficiently datum and with sufficient precision to allow colonies high spectral resolution to allow the physical and sampling locations to be revisited.

processes responsible for the reflectance behaviour to be identified. With more detailed and less ambiguous knowledge of the optical properties of penguin guano it should be possible to recognise it more reliably in a much wider range of types of imagery, thus extending the scope for identifying previously unknown colonies and for measuring those that are already known. A secure understanding of the factors that control its optical properties should give a better chance of understanding the range of variation that can occur, and scope for developing more robust methods for identifying penguin colonies from remotely sensed imagery. Our principal focus is on the 'pinking-up' of Adélie guano, although we also investigate the guano of the other Pygoscelis species to determine whether it is likely that they can be discriminated from one another (and potentially also from other seabird species) (Fretwell et al. 2015, LaRue \& Knight 2014, Lynch \& LaRue 2014, Schwaller et al. 2013). As Pygoscelis penguins all consume krill, to a greater or lesser extent, their guano generally has a similar coloration. Therefore at some sites on the Antarctic Peninsula and the southern Scotia Arc, where the three species breed sympatrically (Waluda et al. 2014) distinguishing which species are present using remote sensing methods is challenging. We consider the question of whether the transition from predominantly white guano to pink can be reliably identified.

\section{Methods}

We collected in situ reflectance spectra, at high spectral and spatial resolution, from Signy Island, in

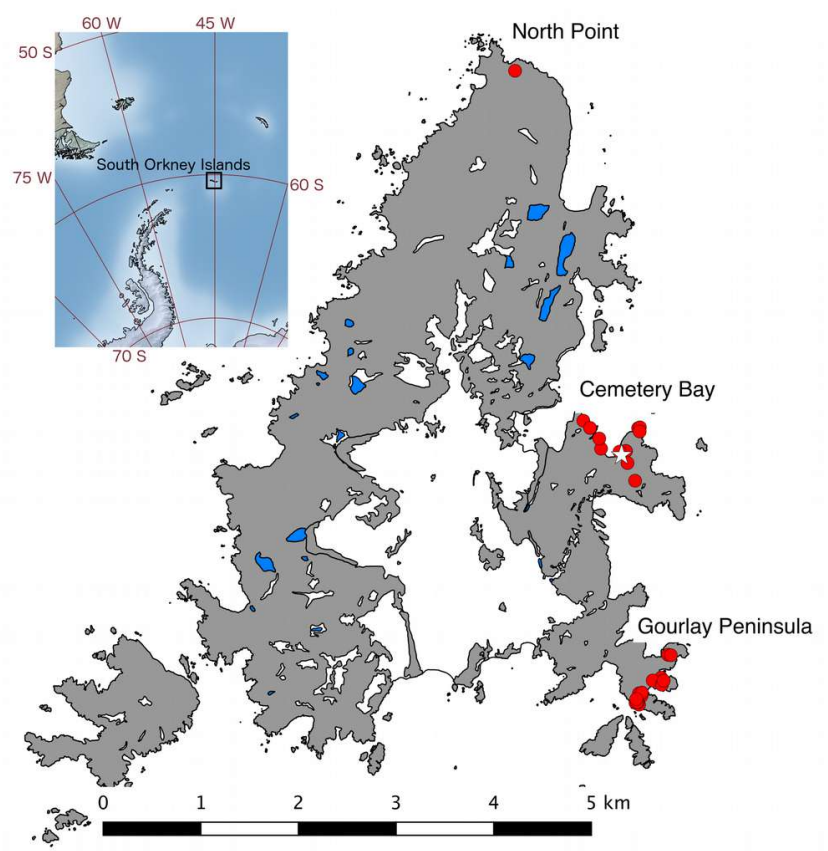

Fig. 1. Location of Signy Island and measurements sites (red dots). The white star indicates the position of the

BAS research station. (Large-scale map data from Antarctic Digital Database: http://www.add.scar.org)

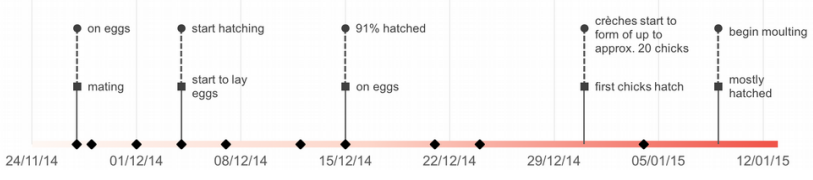

Fig. 2. Timeline showing data collection (diamonds) and key events for Adélies (circles) and chinstraps (squares).

The bar at the bottom represents schematically the changing colour of guano. 
Our sampling strategy was determined by our aim of collecting single-sample spectra covering the full range of spectral diversity of penguin guano as it evolved over time. It was not our intention to follow the spectral evolution of specific colonies over time, and it would not have been feasible to collect representative samples from the same colony at regular intervals. Our ability to access specific colonies was restricted by weather conditions, and our ability to move freely within colonies was restricted by the requirement not to disturb the penguins unnecessarily. Instead, we attempted as best we could to obtain spectra from samples that we judged, by eye, to be representative of any colony we visited at the time we visited it. We collected data from 68 guano samples, representing different Pygoscelis species, guano colours, stages of the breeding cycle, and environmental conditions. Almost half of the samples were obtained from a single Adélie colony.

We used a Fieldspec Pro field-portable spectroradiometer (ASD Inc.) to collect spectral data. This instrument detects radiation in the range 350$2500 \mathrm{~nm}$, using three separate detection subsystems responding to the visible and near infrared (VNIR: 350-970 nm), short-wave infrared 1 (SWIR1: 970$1750 \mathrm{~nm}$ ) and short-wave infrared 2 (SWIR2: 1750$2500 \mathrm{~nm})$ ranges. The spectral resolution of the instrument is around $3 \mathrm{~nm}, 10 \mathrm{~nm}$ and $10 \mathrm{~nm}$, respectively, in these three ranges. The 'bare fibre' fore-optic of the Fieldspec Pro was used, giving a field of view of approximately $25^{\circ}$ radius (typically $0.4 \mathrm{~m}$ at $0.8 \mathrm{~m}$ observing distance), which was normally sufficient to ensure that a homogeneous target material was being measured. For small samples the observing distance was reduced to approximately $0.1 \mathrm{~m}$, giving a field of view of radius ca $0.05 \mathrm{~m}$. Reflectance values were subsequently calculated using measurements of a calibrated 'Spectralon' white panel reference which replaced the target material in the same geometrical configuration (fig. 3). A set of measurements for a single sample consisted of at least three replicates (sample and white panel reference), together with a digital photograph of the sample. Because it was impossible to remove most guano samples for analysis in the laboratory, we relied on daylight to provide the illumination needed to make measurements in situ. It was important to ensure that illumination conditions remained constant during a set of measurements (i.e. for typically around 5 minutes), and since conditions of continuous direct sunlight are not common on Signy Island this was usually not easy to achieve. We made frequent observations of sky conditions and monitored the intensity of incident light using a luxmeter.

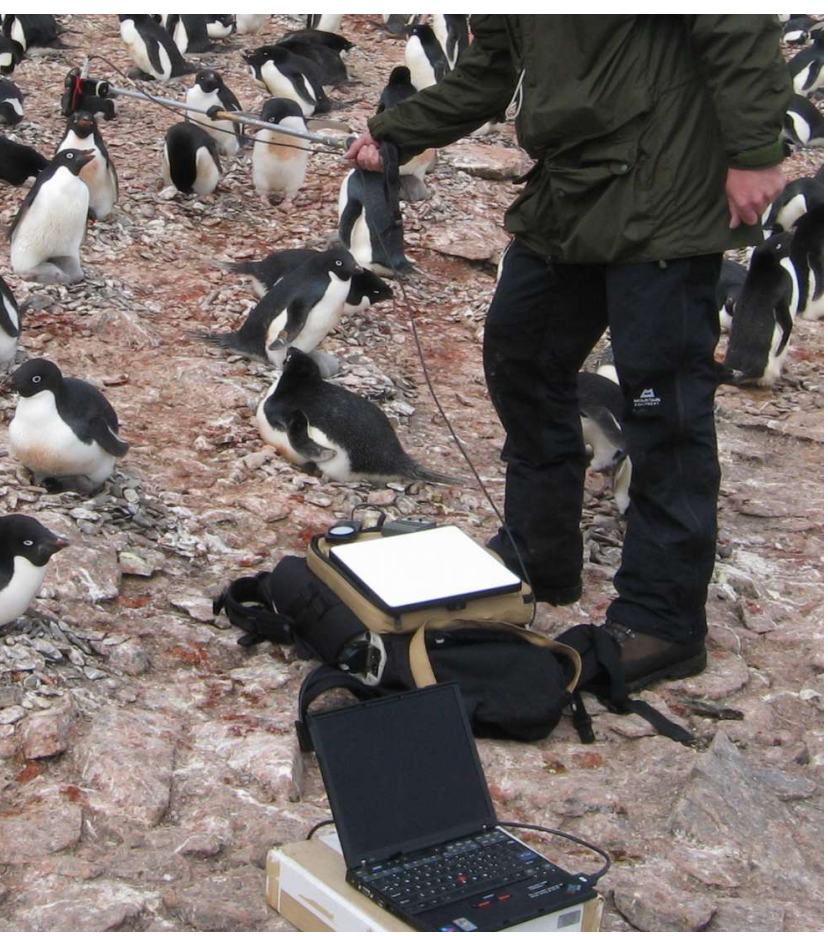

Fig. 3. Data collection. The sensor head is supported on a pole and alternately views the target and the

Spectralon calibrator. The optical signal is carried by an optical fibre to the spectrometer (underneath Spectralon panel), which is controlled by the laptop computer.

In addition to samples of penguin guano, we also took measurements from samples of Adélie penguin vomit, i.e. the largely undigested stomach contents being carried by parents to feed chicks. Ten samples had been collected as part of a long term monitoring project following CCAMLR Ecosystem Monitoring Programme standard procedures (CCAMLR 1992) and made available to us for our spectral measurements after analysis of constituents had been carried out. Two very distinct types of vomit were observed: grey, predominantly fish-based, and pink, predominantly krill-based. We made our spectral measurements on these samples within at most three days of their collection, the samples having been frozen (and then thawed immediately prior to measurement) in the meanwhile.

We calculated the reflectance spectra for 62 of our 68 guano samples (the other six were not usable because of low light levels) and 10 vomit samples using programs written in the GNU Octave language (Eaton et al. 2015). In all cases it was impossible to retrieve meaningful spectra in the range 1830-1900 nm, and in most cases (where the incident light level was below around $75 \mathrm{klx}$ ) the range 1350$1400 \mathrm{~nm}$ was also impossible to retrieve, owing to the effect of atmospheric water vapour removing absorbing all of the daylight illumination in these parts of the spectrum (Rees 2013).

In order to try to identify a number of 
'generic' spectra from our set of 62 spectra, we generalised them as follows. First, we edited the spectra to include only the wavelength ranges 350 1350 and 1400-1800 nm; other wavelengths, including the SWIR2 band, were deemed to be too strongly affected by atmospheric water vapour absorption for the spectra to be reliable. Next, we reduced the data volume by averaging the spectra into 10-nm bins i.e. 350-359, 370-369, ... 1340-1349, $1400-1409, \ldots 1790-1799 \mathrm{~nm}$, so that each sample was represented by 140 variables. We then used a statistical clustering method (agglomerative hierarchical clustering, using five different agglomeration methods and the Euclidean distance metric, implemented in the statistical package SPSS) to combine similar spectra into groups, allowing the method to guide the choice of the final number of groups. We also inspected the dendrogram, showing dissimilarities between spectra, to help choose the final number of groups into which the spectra should be combined. Possible associations between the groups and, firstly, penguin species and, secondly, guano colour and condition were investigated by calculating Cramér's V statistic (Cramér 1999).

\section{Results}

We collected most spectra $(n=31)$ from a single, large Adélie colony centred at $60.7312 \mathrm{~S}, 45.5884 \mathrm{~W}$. Further Adélie spectra $(n=9)$ were collected from a second colony centred at $60.7267 \mathrm{~S}, 45.58334 \mathrm{~W}$. Chinstrap spectra were collected from colonies centred at $60.7295 \mathrm{~S}, 45.5841 \mathrm{~W}(n=2), 60.7304 \mathrm{~S}$, $45.5880 \mathrm{~W}(n=4)$ and $60.7286 \mathrm{~S}, 45.5838 \mathrm{~W}(n=6)$. The remaining spectra were collected as indicated in fig. 1. The visual appearance of most samples was white or pink, though some other colours were noted and measured, including green and, especially in the case of gentoo penguins, yellow.

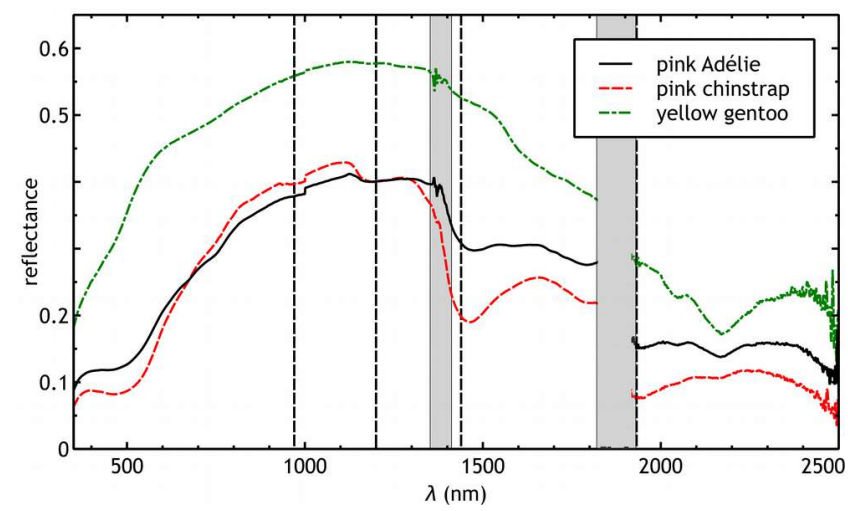

Fig. 4. Full-range spectra: average spectra for pink Adélie and Chinstrap, and yellow Gentoo, guano. Dashed lines show absorption peaks of liquid water; grey bars show regions of the spectrum in which the atmosphere is particularly opaque as a result of water vapour absorption. The range from 1830-1900 nm was never measurable for this reason.

Only those few guano spectra collected when the ambient illumination exceeded around $75 \mathrm{klx}$ gave adequate representation of the entire spectral range, including the SWIR2 region, accessible by the FieldSpec Pro instrument. These spectra reveal an absence of any narrow spectral features, but do show some differences in the SWIR2 region (fig. 4). There are broad absorption features, though not present in all the spectra, at around 550, 1450 and $2150 \mathrm{~nm}$. We were unable to calculate meaningful spectra for the vomit samples in the SWIR2 range, but the spectra in the range $350-1800 \mathrm{~nm}$ again revealed only broad spectral features (fig. 5). The spectra of krill- and fish-based vomit are essentially identical in the range 1100-1800 nm and have common absorption features above about $800 \mathrm{~nm}$. Broad absorption features due to liquid water were very apparent around 950 and 1450 $\mathrm{nm}$. In the VNIR region the spectra are substantially different, the krill-based vomit being generally more reflective except around $500 \mathrm{~nm}$. The sharp increase in reflectance between 500 and $700 \mathrm{~nm}$ is responsible for the visually strong red coloration. The high reflectance of the krill-based vomit sample between around 700 and $1200 \mathrm{~nm}$ is very likely to be due to multiple scattering from undigested particles of chitin.

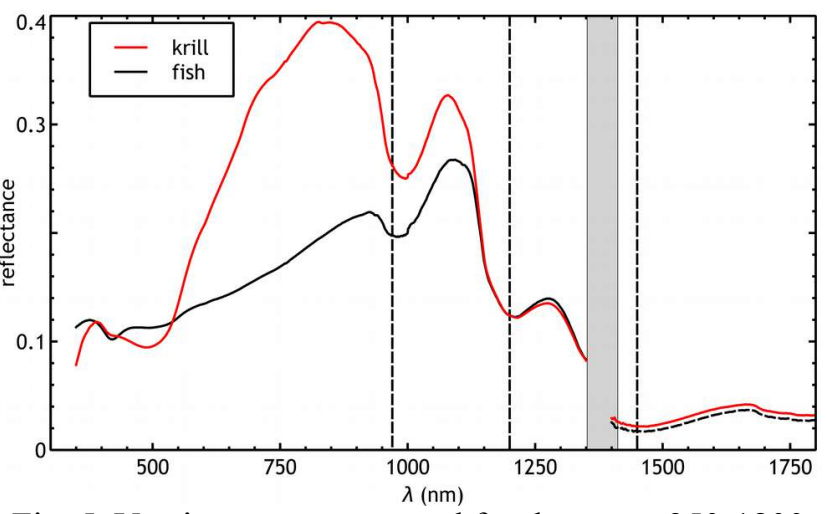

Fig. 5. Vomit spectra measured for the range 350-1800 $\mathrm{nm}$, excluding the range 1350-1400 $\mathrm{nm}$. Dashed lines show absorption peaks of liquid water; the grey bar

shows the region of the spectrum in which the atmosphere is particularly opaque as a result of water vapour absorption. 


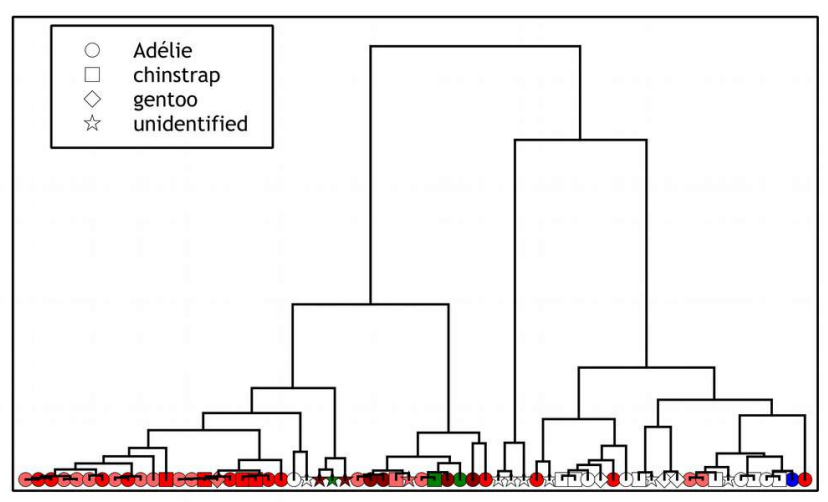

Fig. 6. Dendrogram from between-group hierarchical clustering. (Four other clustering methods were used, giving slightly different histograms.) The shapes of the symbols denote the species, while the colours represent

the visual appearance of the guano: white (or light yellow), pink, red, red/wet (shown as dark red), green, or mixed (shown as blue).

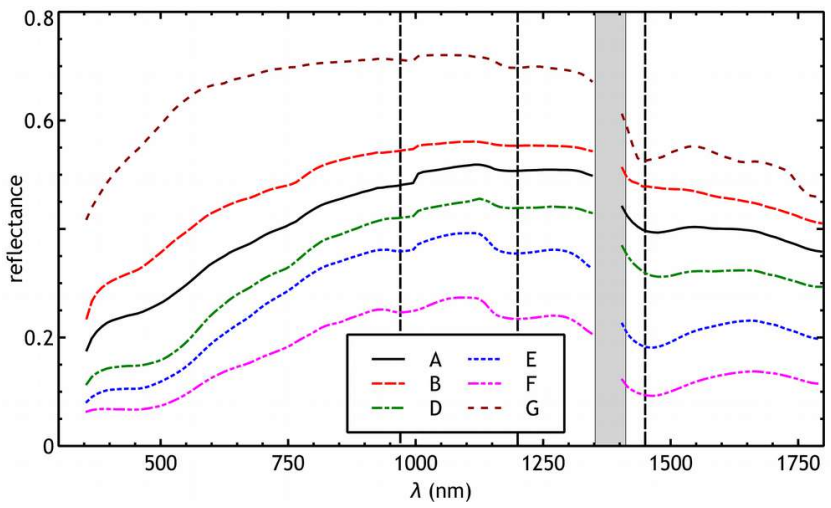

Fig. 7. Six average group spectra defined by clustering 62 spectra of penguin guano. The range $1350-1400 \mathrm{~nm}$ is excluded. Dashed lines show absorption peaks of

liquid water; the grey bar shows the region of the spectrum in which the atmosphere is particularly opaque as a result of water vapour absorption.

\section{Generalisation of the 62 guano specta produced a thests a weak feature around 1150 nin, and that} Generalisation of the 62 guano spectra produced a there is a feature in the SWIR2 region, at around 2200 reasonably clear indication that they could be $\mathrm{nm}$ ). Narrow spectral features are not present, and this combined into at most seven groups (fig. 6), which suggests that high spectral resolution measurements we designated A to G. We calculated the average may not be needed to identify penguin guano. The spectra of six of these seven groups (one of them - absorption feature around $550 \mathrm{~nm}$ is proposed to be group $\mathrm{C}$ - only had a single member and was not due to the pigment astaxanthin, known to be included in subsequent analyses). These average responsible for the pink coloration of krill and other spectra again exhibited only broad spectral features, species (Auerswald et al. 2008, Dissing et al. 2011, but were clearly differentiable on the basis of both Fox 1955). To verify this, fig. 8 shows the difference average reflectance and depth of some spectral between pink (group D) and white (average of groups features (fig. 7). Statistical analysis using Cramér's V A and B) guano reflectance plotted over the range test showed that group membership is only weakly 350-1350 nm, together with the experimentally associated with penguin species, but it is more determined reflectance spectrum of astaxanthin strongly associated with the colour and environmental (Dissing et al. 2011). We established the state (whether wet or dry) of the guano.
This study provides the first in-situ well-defined gentoo penguins. We easily observed the three general that are expected depending on food source (Myrcha
the \& Tatur 1991). These spectra show a well-represented change from white(ish) to pink during the breeding Pink guano is expected to arise from krill diets, white from fish diets and green guano from either and so not feeding (Heine \& Speir 1989). The presence of yellow guano in gentoo colonies, which was also accompanied by white and pink deposits, was surprising and may merit future investigation.

We interpret the generic spectra, represented identified as white or light-coloured, dry samples of another. Their spectral shapes are very similar (fig. 7)
and and are probably not truly distinct from one and the differences are probably due to a difference in lower reflectance. D is a group of dry, but rather pinker, guano samples, with a pronounced dip around $\mathrm{D}$ but with a rather stronger water absorption feature around $1450 \mathrm{~nm}$, and $\mathrm{F}$ is more extreme again: these increasing degrees of wetness. Finally the very distinct group $\mathrm{G}$ corresponds to optically thicker white or light-coloured samples.

These six averaged spectra emphasise the importance of broad absorption features centred around $550 \mathrm{~nm}$ and $1450 \mathrm{~nm}$ in controlling differences between guano spectra (fig. 4 also correspondence between the two reflectance scales empirically. Although the two spectra differ in detail, there is good general correspondence in shape and location, lending support to the idea that the main reflect 
principle responsible for the pink appearance of while almost all samples that we collected after this guano samples in this part of the spectrum is the date showed higher values of the redness index. We presence of this pigment. This result, which is suggest that this pattern, which we also observed unsurprising, is also supported by comparison (though at a later date) for the chinstrap penguin between figures 4 and 5 .

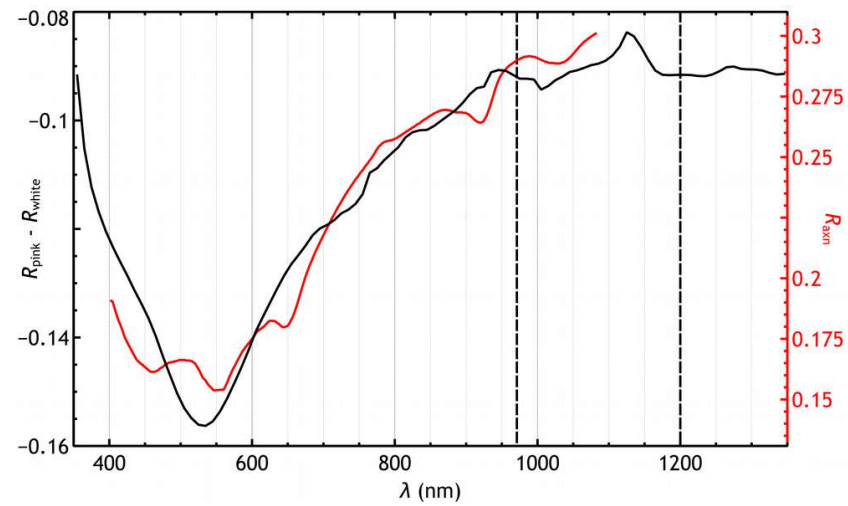

Fig. 8. Published reflectance of astaxanthin (red: scale on right) compared with difference between pink and white guano determined in this work (black: scale on left). Dashed lines show absorption peaks of liquid water. guano, could be used to quantify the phenomenon of 'pinking up' of guano. Variation in penguin breeding phenology is known to occur (Black 2016), including between separate sub-colonies of the same species at the same location (PN Trathan pers. obs. Monroe Island 2015/2016), and this quantitative approach, applied to suitable multispectral remote sensing imagery, could allow variability in breeding phenology to be investigated. The 'wetness' index of nearly all samples is below around 0.25 , except for the measurements obtained on 21 December when much higher values were noted. These samples were all obtained from sites on the Gourlay Peninsula, a couple of days after snowfall which had partially melted. The effect of liquid water on the distribution and appearance of the guano was very marked on this occasion; it resembled a waterlogged red clay soil, or a very thick beetroot soup. Despite the very high values of the wetness index from these measurements, the redness index was within the normal range,

The other broad spectral features noted in figure 5 confirming the ability of the two indices to separate correspond well with the absorption maxima of liquid the effects of the pinking-up and environmental water at around 970, 1200 and $1450 \mathrm{~nm}$ (fig. 3 also processes in controlling the guano reflectance. It is shows evidence of a water absorption feature around important to note here that these indices have been $1930 \mathrm{~nm}$ ). Of these, the most prominent is at 1450 derived from measurements derived from single $\mathrm{nm}$. On this basis we propose two simple indices for homogeneous samples of guano. Calculating the same distinguishing between different types and conditions indices for the pixels of a satellite image will invoke of guano: a wetness index and a redness index. In the 'mixed pixel' problem, which is likely to increase terms of the Landsat-8 OLI bands, the wetness index the extent to which the indices vary over time as the is defined as

$$
\frac{r_{5}-r_{6}}{r_{5}+r_{6}}
$$

coverage of guano, both as an areal percentage and as thickness, increases.

Although our results do not suggest that it is possible to discriminate between Pygoscelis penguin

while the redness index is defined as

$$
\frac{r_{4}-r_{3}}{r_{4}+r_{3}}
$$

species on the basis of the spectral properties of their guano alone, the spectrally distinctive redness signal suggests that it may be possible to deduce the phenological stage from suitable imagery and possibly hence possibly to infer the species. Our observations of the visual differences between chinstrap and Adélie colonies, at a time when most Adélie chicks had hatched but most chinstrap chicks The wavelength ranges of these bands are 3: 530-590 had yet to do so, certainly suggest that this should be $\mathrm{nm}$; 4: $640-670 \mathrm{~nm} ; 5: 850-880 \mathrm{~nm} ; 6: 1570-1650$ possible, and use of the wetness index should reduce $\mathrm{nm}$. The values of these two indices for the six possible ambiguities in the interpretation of guanoaverage spectra shown in fig. 7 are as shown in table dominated spectra. It is possible that the difference i. As a result of the limitations on systematic sampling between Adélie and chinstrap colonies might decrease noted earlier, it is not possible to make a useful in subsequent weeks as the chinstrap chicks grow, so statement about trends in these indices over time. further measurements would be beneficial.

However, we can cautiously identify some tendencies in the Adélie data, for which we have the largest here represent a relatively simple approach to number of measurements. extracting physically or physiologically meaningful

None of the samples that we collected in late data from spectra. They can probably be extended. November showed a redness index higher than 0.1 , There is perhaps a suggestion in the group spectra 
presented in fig. 7 that the astaxanthin absorption imagery and that allow the influences of astaxanthin feature broadens as the pigment concentration and environmental conditions to be separated. The increases. If so, this could potentially be exploited by ability to extract a separate 'redness' signal has the examining derivatives of the spectral reflectance potential to allow the 'pinking-up' process to be functions (which should therefore not be smoothed to tracked over time in a series of images, which would $10 \mathrm{~nm}$ spectral resolution). Both indices could help to distinguish between species, although in perhaps be standardised between different guano practice this ability will be compromised by the types, although that would require more data than difficulty of obtaining a time-series of images from a were collected for this work. location such as Signy with very frequent cloud

We can ask what more would be needed to cover. The data collected during the fieldwork for this develop the results presented here into a robust research did not reveal any systematic differences algorithm for identifying Antarctic penguin colonies between the spectra of guano from different penguin and estimating the number of birds within them. That species, which is probably unsurprising in view of the remains a difficult goal to reach, though it is nearer. very marked effect of diet on the spectra. Taken At least for krill-feeding species where the together, these results should significantly enhance astaxanthin signal in the pink guano can be our ability to recognise penguins from guano stains in recognised, the major difficulties will be obtaining satellite images and understand the potential imagery at sufficiently high spatial resolution and at differences in coloration of guano.

the right time of year, and understanding better the

spatial characteristics of penguin colonies - including Acknowledgements

variations in packing densities. Future work should

focus on determining how much guano spectra The fieldwork necessary for this work was supported influences colony spectra (what extent of spatial by British Antarctic Survey through a Collaborative mixing is there) as well as what level of spatial Gearing Scheme award CGS-97 to Gareth Rees and resolution is necessary for accurate colony Phil Trathan, and the ASD Fieldspec Pro was made examination especially given the high costs of higher available through an award (ref. 696.0614) from the resolution imagery and the need for low cost penguin UK Natural Environment Research Council (NERC) counts.

\section{Conclusions}

Field Spectroscopy Facility. We acknowledge with gratitude the support and companionship of the entire staff of the BAS research station at Signy, and especially of Matt Jobson, the Base Commander. We

The fundamental aim of this research was to define gratefully acknowledge also the input of Stacey 'generic' spectra for the guano of Pygoscelis penguins Adlard, who was generous in sharing her at very high spectral and spatial resolution, and this understanding of Signy's penguin population and her has been accomplished. These data will provide a samples of penguin vomit. Jennifer Brown is baseline for the development of improved methods supported by a NERC PhD studentship for detecting and analysing Antarctic penguin NE/L501633/1. We gratefully acknowledge the colonies. As has been noted and exploited in previous constructive criticisms of a number of reviewers, work, breeding colonies 'pink up' as the diet shifts to which have materially improved this paper.

one rich in krill. The reason for this pink coloration is

unambiguously, if unsurprisingly, attributable to the Data availability

pigment astaxanthin responsible for the pinkness of

the krill itself. Apart from the presence of this Processed reflectance spectra and accompanying pigment, the other main factor controlling the optical sample photographs will be available at the properties of the majority of penguin guano at this University of Cambridge research repository stage in the breeding cycle is environmental (https://doi.org/10.17863/CAM.8273) from 1 January processing through waterlogging. We propose two 2018.

simple mathematical indices, based on reflectance

values in the red and near infrared parts of the

spectrum, that can be calculated from satellite 


\section{References}

Agnew, D. 1997. The CCAMLR Ecosystem Monitoring Programme. Antarctic Science, 9, $235-242$.

AINLEY, D. 2002. The Adélie penguin: bellwether of climate change. New York: Columbia University Press, 310 pp.

Auerswald, L., Freier, U., LopatA, A. \& MeYer, B. 2008. Physiological and morphological colour change in Antarctic krill, Euphausia superba: a field study in the Lazarev Sea. Journal of Experimental Biology, 211, 3850-3858.

BLACK, C. 2016. A comprehensive review of the phenology of Pygoscelis penguins. Polar Biology, 39, 405-432.

Boersma, P. 2008. Penguins as marine sentinels. Bioscience, 58, 597-607.

CCAMLR. 1992. CCAMLR Ecosystem Monitoring program (CEMP) standard methods. Hobart: Commission for the Conservation of Antarctic marine living organisms.

CRAMÉR, H. 1999. Mathematical methods of statistics. Princeton: Princeton University Press.

Croxall, J. \& KIRKWOOD, E. 1979. The distribution of penguins on the Antarctic Peninsula and islands of the Scotia Sea. Cambridge: British Antarctic Survey.

Dissing, B., Nielsen, M., ERsbØLl, B. \& Frosch, S. 2011. Multispectral imaging for determination of astaxanthin concentration in salmonids. PLOS ONE, 10.1371/journal.pone.0019032.

EAton, J., BAteman, D., HAuberg, S. \& Wehbring, R. 2015. GNU Octave version 4.0.0 manual: a high-level interactive language for numerical computations. Available at:

http://www.gnu.org/software/octave/doc/interpreter/.

Forcada, J. \& Trathan, P. 2009. Penguin responses to climate change in the Southern Ocean. Global Change Biology, 15, 1618-1630.

Fox, D. 1955. Astaxanthin in the American Flamingo. Nature, 175, 942-943.

Fretwell, P., Phillips, R., Brooke, M., Fleming, A. \& McArthur, A. 2015. Using the unique spectral signature of guano to identify unknown seabird colonies. Remote Sensing of Environment, 156, 448-456.

Fretwell, P. \& TRAThan, P. 2009. Penguins from space: faecal stains reveal the location of emperor penguin colonies. Global Ecology and Biogeography, 18, 543-552.

HeIne, J. \& SPEIR, T. 1989. Ornithogenic soils of the Cape Bird Adelie penguin rookeries, Antarctica. Polar Biology, 10, 89-99.

LARUE, M. \& KNIGHT, J. 2014. Applications of Very High-Resolution imagery in the study and conservation of large predators in the Southern Ocean. Conservation Biology, 28, 1731-1735.

LaRue, M., Lynch, H., Lyver, P., Barton, K., Ainley, D., Pollard, A., Fraser, W. \& Ballard, G. 2014. A method for estimating colony sizes of Adélie penguins using remote sensing imagery. Polar Biology, 37, 507-517.

LYNCH, H. \& LARUE, M. 2014. First global census of the Adélie penguin. The Auk, 131, 457-466.

LyNCH, H., WhITE, R., BLACK, A. \& NAVEEN, R. 2012. Detection, differentiation, and abundance estimation of penguin species by high resolution satellite imagery. Polar Biology, 35, 963-968.

Myrcha, A. \& TATUR, A. 1991. Ecological role of the current and abandoned penguin rookeries in the land 
environment of the maritime Antarctic. Polish Polar Research, 12, 3-24.

Naveen, R., LynCh, H., Forrest, S., Mueller, T. \& Polito, M. 2012. First, complete site-wide penguin survey at Deception Island, Antarctica reveals massive declines consistent with climate change. Polar Biology, 35, 1879-1888.

ReEs, W. 2013. Physical Principles of Remote Sensing. 3rd ed. Cambridge: Cambridge University Press, 441 pp.

SCHWAller, M., Benninghoff, W. \& Olson, C. 1984. Prospects for satellite remote-sensing of Adélie penguin rookeries. International Journal of Remote Sensing, 5, 849-853.

SChwaller, M., Olson, C., MA, Z., ZhU, Z. \& DAHMER, P. 1989. A remote-sensing analysis of Adélie penguin rookeries. Remote Sensing of Environment, 28, 199-206.

SCHWAller, M., SOUthwell, C. \& EMMERSON, L. 2013. Continental-scale mapping of Adélie penguin colonies from Landsat imagery. Remote Sensing of Environment, 139, 353-364.

Southwell, C., Emmerson, L., McKinlay, J., Newbery, K., Takahashi, A., Kato, A., Barbraud, C., DELORD, K. \& WEIMERSKIRCH, H. 2015. Spatially extensive standardized surveys reveal widespread, multi-decadal increase in East Antarctic Adélie penguin populations. PLoS ONE, 10.

Trathan, P., García-Boroboglu, P., Boersma, D., Bost, C.-A., Crawford, R., Crossin, G., Cuthbert, R., et al. 2015. Pollution, habitat loss, fishing, and climate change as critical threats to penguins.

Conservation Biology, 29, 31-41.

TRATHAN, P.N. 2004. Image analysis of color aerial photography to estimate penguin population size. Wildife Society Bulletin, 32, 332-343, 10.2193/0091-7648(2004)32[332:IAOCAP]2.0.CO;2.

Waluda, C., Dunn, M., Curtis, M. \& Fretwell, P. 2014. Assessing penguin colony size and distribution using digital mapping and satellite remote sensing. Polar Biology, 37, 1849-1855.

Weimerskirch, H., Inchausti, P., Guinet, C. \& Barbraud, C. 2003. Trends in bird and seal populations as indicators of a system shift in the Southern Ocean. Antarctic Science, 15, 249-256.

Witharana, C. \& LYNCH, H.J. 2016. An Object-Based Image Analysis Approach for Detecting Penguin Guano in very High Spatial Resolution Satellite Images. Remote Sensing, 8, 375, 10.3390/rs8050375.

WOEHLER, E. \& RIDDLE, M. 1998. Spatial relationships of Adélie penguin colonies: implications for assessing population changes from remote imagery. Antarctic Science, 10, 449-454. 